\title{
As representações sociais da depressão em adolescentes no contexto do ensino médio'
}

\author{
Depression social representation \\ in the high school context
}

\author{
Airton Pereira do Rêgo BARROS ${ }^{2,5}$ \\ Maria da Penha de Lima COUTINHO 3,5 \\ Ludgleydson Fernandes ARAÚJO 4,5 \\ Alessandra Ramos CASTANHA ${ }^{4,5}$
}

\begin{abstract}
Resumo
Este estudo objetivou verificar as representações sociais da sintomatologia da depressão em adolescentes no contexto do ensino médio de escolas públicas e privadas da cidade de João Pessoa, PB. Participaram da amostra 60 adolescentes (54\% do sexo feminino e 46\% do masculino) com média de idade de 16 anos, com a sintomatologia depressiva. Utilizaram-se o Children Depression Inventory como instrumento de Screening e entrevistas semi-estruturadas aplicadas de forma individual. As entrevistas foram analisadas por meio da técnica de análise de conteúdo temática. Os resultados apontam as seguintes categorias empíricas: concepção/descrição, causas e tratamento, essas subdivididas em subcategorias que enfatizaram principalmente os aspectos afetivos, cognitivos, psicossociais e comportamentais. Percebe-se que os grupos constroem suas representações não apenas baseados no conhecimento erudito ou nas comunicações informais, mas também no conjunto de problemas práticos que encontram no âmbito sociocultural diretamente inter-relacionados à pertença e à identidade grupal.
\end{abstract}

Palavras-chave: adolescentes; depressão; representações sociais.

\begin{abstract}
This study's proposal was to verify the social representations of the depression's symptoms in adolescents in high school context from public and private schools, in Joäo Pessoa city, PB. Sixty adolescents with depression's symptoms from both genders (54\% female and $46 \%$ male), whose ages were around 16, have participated in this study. It was utilized the Children Depression Inventory as the screening instrument, as well as semi-structured interviews, which were applied individually. The interviews were analyzed according to the thematic content technique. The results pointed out the following empirical categories: conception/description, causes and treatment. These categories were further divided in subcategories that had mainly emphasized the affective, cognitive, psycho-socials and behavioral aspects. It was realized the groups not only construct their representations based on the erudite knowledge or the informal communications, but also based on the set of practical problems that they have faced in the socio-cultural scope, which are directly interrelated to the group identity and acceptance.
\end{abstract}

Key words: adolescents; depression; social representations.

$\boldsymbol{\nabla} \mathbf{V}$

1 Artigo elaborado a partir da dissertação de A.P.R. BARROS, intitulada "As representações sociais da depressão em adolescentes no contexto escolar". Programa de Pós-Graduação em Psicologia Social, Universidade Federal da Paraíba, 2005.

2 Professor, Instituto de Psicologia, Universidade Federal de Uberlândia. Av. Engenheiro Diniz, 1178, 38400-902, Uberlândia, MG, Brasil. Correspondência para/ Correspondence to: A.P.R. BARROS. E-mail:<airtonpsocial@yahoo.com.br>.

3 Professoras Doutoras, Departamento de Psicologia, Programa de Pós-Graduação em Psicologia Social, Universidade Federal da Paraíba. João Pessoa, PB, Brasil.E-mail:<penhalcoutinho@bol.com.br>.

4 Mestrandos em Psicologia Social, Programa de Pós-Graduação em Psicologia Social, Universidade Federal da Paraíba. João Pessoa, PB, Brasil.

5 Núcleo de Pesquisa Aspectos Psicossociais de Prevenção e Saúde Coletiva, Universidade Federal da Paraíba. João Pessoa, PB, Brasil.

Agradecimentos: Os autores agradecem o apoio financeiro da CAPES para realização desta pesquisa. 
A depressão tem se configurado como um dos mais graves problemas de saúde coletiva da atualidade, com índices elevados da doença, que ocorre em pessoas de qualquer idade e condição socioeconômica, independentemente das culturas e tradições, conferindo-lhe o status de fenômeno global (Widlöcher \& Hardy, 1991; Coutinho, Gontiés, Araújo \& Sá, 2003). De acordo com Coutinho (2001), o largo espectro de alcance da doença se deve ao fato de ela se apresentar como um estado grave ao longo do desenvolvimento histórico-sociocultural do sujeito frente à saúde e à doença.

Na década de 1990, a síndrome foi estimada como a quarta causa específica de incapacitação, através de uma escala global para comparação entre várias doenças. A previsão para o ano de 2020 é a de que será a segunda causa em países desenvolvidos e a primeira em países em desenvolvimento (Murray \& Lopez, 1997; Bahls, 2002a). Quando comparada às principais condições médicas crônicas, a depressão só tem equivalência, em incapacitação, às doenças isquêmicas cardíacas graves (Bahls, 2002a).

O reconhecimento oficial da depressão em crianças e adolescentes é recente, conseqüentemente, os estudos científicos a esse respeito são ainda carentes de avanços. Foi a partir de 1975 que o National Institute of Mental Health of the US (NIMH) reconheceu a existência da depressão em crianças e adolescentes (Bahls, 2002b). O reconhecimento da patologia no grupo infanto-juvenil ocasionou, nas décadas de 1970 e 1980, um avanço no desenvolvimento de entrevistas diagnósticas estruturadas, semi-estruturadas e escalas de avaliação da depressão (Coutinho, 2001). Apesar desses avanços, é por meio do estudo de Kovács e Beck (1977) que a depressão infanto-juvenil passa a ser considerada de fato uma síndrome independente da observada na fase adulta, estabelecendo-se, a partir daí, critérios de diagnóstico clínico, biológico e psicossocial.

Vários autores têm chamado a atenção para a ocorrência do fenômeno da depressão nessa faixa etária, que parece ser a mais afetada (Brent, 1993; Birmaher, Ryan, Williamson, Brent, Kaufman \& Dahl, 1996; Coutinho, 2001). No estudo Los Angeles Epidemiologic Catchment Area Project, verificou-se que 25\% dos adultos com depressão maior relataram o primeiro episódio da doença tendo ocorrido antes dos dezoito anos de idade (Olsson \& Von Knorring, 1999).

Considera-se que a depressão na infância e na adolescência apresenta natureza duradoura, afetando múltiplas funções e causando significativos danos psicossociais (Bahls, 2002b). É, também, uma doença debilitante e recorrente, envolvendo um alto grau de morbidade e mortalidade, e isto é de grande interesse para a saúde coletiva, embora, ainda, freqüentemente, não seja reconhecida nem tratada adequadamente (Mirza \& Michael, 1996; Walter, 1996).

Com relação à apresentação dos sintomas nessa fase do desenvolvimento, Kazdin e Marciano (1998) afirmam que mais de $80 \%$ dos jovens deprimidos apresentam humor irritado e perda de energia, apatia e desinteresse, retardo psicomotor, sentimentos de desesperança e culpa, perturbações do sono principalmente hipersonia -, alterações de apetite e peso, isolamento e dificuldade de concentração.

Em pesquisa sobre sintomas de depressão em adolescentes entre quatorze e dezoito anos de idade, em amostra comunitária no Oregon Adolescent Depression Project, nos EUA, Roberts, Lewinsohn e Seeley (1995) encontraram, como sintomas mais prevalentes, o humor deprimido, alterações do sono e dificuldades no pensamento (problemas de concentração e pensamento negativista); e como sintomas mais estáveis, o humor deprimido e anedonia. Outras características próprias dessa fase são o prejuízo no desempenho escolar, a baixa auto-estima, as idéias e tentativas de suicídio e graves problemas de comportamento, especialmente, o uso abusivo de álcool e drogas (Martin \& Cohen, 2000; Assumpção Jr \& Kuczynski, 2001).

Destacam-se algumas diferenças entre a manifestação depressiva em adolescentes do sexo feminino e do masculino. As meninas relatam mais sintomas subjetivos, como sentimentos de tristeza, vazio, tédio, raiva e ansiedade; costumam também ter mais preocupação com a popularidade, menos satisfação com a aparência, mais conscienciosidade e menos auto-estima, enquanto os meninos relatam mais sentimentos de desprezo, desafio e desdém, e apresentam problemas de conduta, como falta às aulas, fugas de casa, violência física, roubos e abuso de substâncias psicoativas (Sadler, 1991; Baron \& Campbell, 1993). 
Com o desenvolvimento do pensamento abstrato há uma compreensão mais clara acerca da morte, conseqüentemente, nos adolescentes depressivos, tanto as idéias de suicídio, como as tentativas, alcançam uma dimensão maior. A ocorrência do comportamento suicida entre jovens aparenta estar aumentando nas últimas décadas, e a adolescência destaca-se como o período mais relacionado à morte por causas violentas (Gunnell, 2000; American Medical Association, 2002).

No Brasil, Feijó, Raupp e John (1996), em investigação de comportamento autodestrutivo em adolescentes na faixa de treze a vinte anos de idade, acompanharam, diariamente, em um período de quatro meses, as tentativas de suicídio atendidas pelo Pronto Socorro do Hospital de Clínicas de Porto Alegre, RS. Observaram que 28\% dos casos apresentaram o diagnóstico de depressão e 47\% já haviam tentado o suicídio anteriormente.

Dos primórdios até os dias atuais, a depressão em adolescentes continua suscitando polêmicas, principalmente, quanto à sua especificidade semiológica (Coutinho, 2001). A autora ainda afirma que a palavra depressão é utilizada, no senso comum, de forma genérica e distorcida do seu significado científico, abrangendo uma infinidade de doenças, principalmente as mentais.

As representações sociais constituem formas de conhecimentos socialmente elaborados, que são produzidas pelos grupos de indivíduos para poderem comunicar-se e entender tudo aquilo que Ihes é estranho e não familiar. Nem todo assunto se torna objeto de representação social para os grupos e nem todos os fenômenos que rodeiam o indivíduo são tributários de converterem-se em problemas de pesquisa científica da representação social.

Moscovici (2003) define a representação social como um conjunto de conceitos, afirmações e explicações originadas no decurso do cotidiano, no decurso das comunicações interindividuais. Elas são equivalentes, em nossa sociedade, aos mitos e sistemas de crenças das sociedades tradicionais; elas podem, até mesmo, ser vistas como uma versão contemporânea de senso comum.

As representações sociais são produzidas pelas interações e comunicações no interior dos grupos sociais, refletindo a situação dos indivíduos no que diz respeito aos assuntos que são objeto do seu cotidiano. Nas palavras de Jodelet (2001), as representações sociais são um saber prático. A literatura científica denomina essas teorias, criadas pelos grupos, de teorias do senso comum (Moscovici, 1961). São, justamente elas, o principal objeto de estudo das representações. A função essencial da representação social, para aqueles que representam, é tornar aquilo que não é familiar em algo familiar, próximo e prático (Moscovici, 2003).

A representação social é vista como um processo público de criação, elaboração, difusão e mudança do conhecimento compartilhado no discurso cotidiano dos grupos sociais (Doise, 1990; Jodelet, 2001; Moscovici, 2003). E é compreendida como elaboração de um objeto social pela comunidade com o propósito de conduzir-se e comunicar-se.

Coutinho (2001) lembra que, com o expressivo aumento das pesquisas sobre o fenômeno da depressão, vários são os aportes teóricos que o vêm pesquisando e analisando, uns focalizando mais os aspectos orgânicos, outros os aspectos psicológicos. Porém, em virtude de sua complexidade, e por apresentar-se de forma prolixa e multifacetada, seria reducionismo tentar explicar um problema tão plural dentro de uma ou outra visão específica.

A presença de uma doença na vida de uma pessoa traz alterações significativas no seu organismo, no seu modo de vida e nas relações sociais estabelecidas. A sintomatologia da depressão pode implicar sérias dificuldades, uma vez que a imprecisão diagnóstica pode resultar em fortes seqüelas, privações sociais e, inclusive, em morte.

Para Coutinho (2001), acessar as representações sociais da sintomatologia da depressão é tentar não apenas compreender as formas que os indivíduos utilizam para criar, transformar e interpretar essa problemática vinculada à sua realidade, como, também, conhecer seus pensamentos, sentimentos, percepções e experiências de vida compartilhadas, destacadas nas modalidades diferenciadas de comunicação, de acordo com a classe social a que pertencem e às instituições às quais estão vinculados.

Deve-se procurar perceber como tais representações emergem e suas relações entre si, 
como, também, em que medida uma determina a outra, pois os comportamentos adotados por um indivíduo ou grupo de indivíduos acometidos da sintomatologia depressiva são resultantes do modo como esses a representam socialmente e do significado pessoal que ela adquire em suas vidas.

Sendo assim, o problema de pesquisa que determinou a realização deste estudo foi verificar como os adolescentes que apresentam a sintomatologia depressiva representam a doença e os seus sintomas, assim como comparar os resultados da elaboração da sintomatologia da depressão em adolescentes de instituições públicas e privadas.

\section{Método}

\section{Participantes}

A pesquisa foi desenvolvida em duas escolas, uma pública e outra privada, da cidade de João Pessoa, PB, escolhidas por suas semelhanças estruturais (pela disponibilidade de número significativo de estudantes do ensino médio e a diversidade sociocultural) e pela abrangência sociodemográfica dos sujeitos ali inseridos. A amostra foi composta por 60 adolescentes, $54 \%$ do sexo feminino e $46 \%$ do sexo masculino, na faixa etária entre catorze e dezoito anos, que cursavam da primeira à terceira série do ensino médio e que apresentaram a sintomatologia depressiva, de acordo com o Children Depression Inventory (CDI).

\section{Instrumentos}

\section{Inventário de Depressão Infantil}

O Children's Depression Inventory (CDI) foi elaborado por Kovács (1985). Seu objetivo geral é detectar a presença e a severidade do transtorno depressivo em crianças e adolescentes. É um instrumento adaptado do Beck Depression Inventory (BDI) para adultos. O CDI é adequado para ser aplicado em crianças e adolescentes situados na faixa etária dos sete aos dezessete anos de idade, tendo sido hábil na identificação de alterações afetivas, de humor, de capacidade hedônica, de funções vegetativas e autoavaliativas e de outras condutas interpessoais.
Neste estudo, foi utilizada uma forma do CDI adaptada e normatizada em uma população brasileira (Barbosa, Gaião, Andrade, Dias \& Di Lorenzo, 1995). Na referida versão, o CDI é composto por vinte itens, tendo, cada um, três alternativas de resposta. Cada resposta do instrumento possui um valor correspondente que varia de 0 a $2(a=0 ; b=1 ; c=2)$, sendo o somatório total dos valores das respostas o escore a ser considerado. Seguindo os critérios de validação do instrumento, a pesquisa adotou o ponto de corte 17 para delimitação dos sujeitos com sintomatologia depressiva.

\section{Entrevistas semi-estruturadas}

Utilizaram-se entrevistas semi-estruturadas, constituídas, inicialmente, de itens referentes à identificação sociodemográfica dos participantes, com o intuito de registrar dados como gênero, idade, escolaridade, estado civil dos pais; e uma segunda parte composta por questões que foram formuladas, tendo como pressupostos os objetivos da pesquisa e o estado atual da arte, tais como: fale sobre tudo o que você entende por depressão; descreva o que sente uma pessoa depressiva; a depressão tem cura? Se sim, qual o tratamento?

\section{Procedimento}

Inicialmente, o projeto foi enviado ao Comitê de Ética em Pesquisa com Seres Humanos do Centro de Ciências da Saúde (CCS), da Universidade Federal da Paraíba, com o intuito de averiguar os parâmetros éticos, tendo como base a resolução n 196/96. Recebendo parecer favorável à realização da pesquisa, uma vez que sua operacionalização não traria nenhum dano à saúde física e/ou psíquica dos participantes, entrou-se em contato com a diretoria das instituições escolhidas, onde os pesquisadores apresentaram-se com uma carta contendo os objetivos e a importância do desenvolvimento da pesquisa. Uma vez autorizada a realização do estudo, iniciou-se a coleta dos dados, de acordo com a aplicação do CDI, em todas as classes da primeira a terceira série do ensino médio das referidas instituições de ensino. O CDI foi aplicado em três turmas de primeiro ano ( $n=241)$, três do segundo $(n=228)$ e três do terceiro $(n=204)$, de ambas as instituições, o que contabilizou um total de 673 participantes. 
Após a avaliação do instrumento, observou-se que 60 adolescentes (30 da rede privada e 30 da rede pública) obtiveram somatório superior ao ponto de corte (17 pontos), que, segundo Barbosa et al. (1995), é representativo de sintomatologia depressiva. Em seguida, os adolescentes constituintes da amostra foram submetidos às entrevistas, individualmente, em salas adequadamente escolhidas nos estabelecimentos de ensino. Os dados foram registrados através de gravador, em sessões sem tempo delimitado.

\section{Análise dos dados}

Os dados coletados por meio das entrevistas foram codificados pela análise de conteúdo temática (Bardin, 2002), que visa obter, por procedimentos sistemáticos e objetivos de descrição do conteúdo das mensagens, indicadores que permitam a inferência de conhecimentos relativos às condições de produção/ recepção (variáveis inferidas). Os dados das entrevistas também foram analisados quantitativamente por meio de análises estatísticas descritivas. Foi utilizado o critério de saturação proposto por Sá (1998) no processo de análise e construção das categorias empíricas. Dessa forma a análise é interrompida quando os conteúdos passam a se repetir seguidamente, sem que novos temas sejam observados no discurso dos atores sociais.

\section{Resultados e Discussão}

As representações sociais da sintomatologia da depressão em adolescentes de escolas públicas (G pub) e privadas ( $G$ priv) foram dimensionadas a partir de três categorias e dez subcategorias empíricas, agrupadas por sua constituição temática, de acordo com o nível e o grau de elaboração simbólica, sendo essas nomeadas a partir de suas características mais marcantes.

As categorias são as seguintes: concepção/ descrição, causas, tratamento. Observou-se uma pluralidade de conceitos e elementos associados às dimensões afetiva, psicossocial, cognitiva, comportamental, orgânica e espiritual. Percebe-se também que independentemente da categoria as representações permearam não apenas em torno da depressão, mas da indissociabilidade do que seja depressão, doença e o ser depressivo doente.
Com relação à concepção/descrição, a sintomatologia da depressão foi ancorada a uma diversidade de conceitos que estão associados às dimensões psicológica (30,0\%), psicossocial (20,5\%), afetivo/comportamental (32,0\%) e físico/orgânica (17,5\%), (Tabela 1).

Os dados apontam, nessa categoria, uma convergência entre os grupos no que tange às freqüências das unidades temáticas, principalmente, nas subcategorias psicológica e psicossocial. Na subcategoria fisico/orgânica, os jovens de escolas públicas apresentaram maior freqüência (19,3\%), no entanto, na subcategoria afetivo/comportamental, os jovens de escolas privadas apresentaram um percentual maior (33,3\%). Denota-se que, em ambos os grupos, a subcategoria mais realçada foi a afetivo/comportamental, seguida da subcategoria psicológica.

A subcategoria afetivo/comportamental foi estruturada a partir de um eixo principal, a depressão como sinônimo de solidão e isolamento. Observou-se que tais sentimentos estão associados à depressão como doença e ao ser depressivo, sendo considerados pela maioria como condição comum a todos que sofrem do problema. A solidão é manifestada na sua forma subjetiva, como o que se sente, sendo o isolamento o representante da ação, ou seja, a conseqüência de estar se sentindo na solidão ou o comportamento deliberado de se isolar dos outros, como se pode observar na fala dos atores sociais:

... é solidão... pessoa sozinha no canto...não tem ninguém para conversar... se sentir solitário mesmo no meio da multidão... não gosta de ficar com pessoas... a vontade que dá é se isolar das pessoas (G Pub) .

... é quando a pessoa fica só... se isola do mundo... quando se isola no quarto e não quer que ninguém entre... é ele mesmo que se isola... pessoa sozinha, isolada em si mesmo... (G Priv).

Essas vivências inferem novas representações da própria existência do sujeito e de suas condições existenciais, visto que o estado de isolamento sinaliza uma desestruturação da pessoa por conseqüência do sentimento de desamparo vivenciado nas práticas sociais.

Na segunda subcategoria, a concepção/ descrição psicológica, a depressão foi concebida como sinônimo de tristeza, conforme as falas mencionadas abaixo: 
Tabela 1. Distribuição da categoria concepção-descrição, de suas subcategorias, das freqüências e percentual.

\begin{tabular}{|c|c|c|c|c|c|c|c|}
\hline \multirow{2}{*}{ Categoria } & \multirow{2}{*}{ Subcategorias } & \multicolumn{2}{|c|}{ GPub } & \multicolumn{2}{|c|}{ G Priv } & \multicolumn{2}{|c|}{$\Sigma$} \\
\hline & & $f$ & $\%$ & $f$ & $\%$ & $f$ & $\%$ \\
\hline \multirow{4}{*}{ Concepção/Descrição } & Psicológica & 49 & 29,5 & 54 & 30,5 & 103 & 30,0 \\
\hline & Psicossocial & 34 & 20,5 & 36 & 20,4 & 70 & 20,5 \\
\hline & $\begin{array}{l}\text { Afetivo- } \\
\text { Comportamental }\end{array}$ & 51 & 30,7 & 59 & 33,3 & 110 & 32,0 \\
\hline & Fisíco-Orgânica & 32 & 19,3 & 28 & 15,8 & 60 & 17,5 \\
\hline \multicolumn{2}{|c|}{ Total } & 166 & 100,0 & 177 & 100,0 & 343 & 100,0 \\
\hline
\end{tabular}

... pessoa que não tem felicidade... tá triste... espírito muito sofrido... pessoa que chora muito... pessoa que não pára de chorar ... pai, mãe e irmã chorando (G Pub).

... eu acho que o mais comum é quando a pessoa tá triste ... é uma pessoa que sempre tá triste ... é um mundo de tristeza ... o que ela pensa e o que ela vive é realmente sinônimo de tristeza ... (G Priv).

Denota-se uma estreita ligação entre a forma de psicopatologia e o sentimento de tristeza (humor deprimido), sendo esse considerado, pela maioria dos estudiosos, um sintoma da doença (Roberts, Lewinsohn \& Seeley, 1995; Kazdin \& Marciano, 1998; Bahls, 2002a).

Verificou-se no discurso dos adolescentes, comumente seguido de choros, uma aparente perda do sentido da vida como sinal de uma profunda tristeza, o que indica que, para o saber da vida cotidiana, compartilhado por esses grupos, a depressão é o mesmo que uma tristeza causada por dores existenciais profundas.

O Diagnostic and Statistical Manual of Mental Disorders (DSM-IV) ressalta a tristeza (humor deprimido) como um dos sintomas mais comuns ao estado depressivo, tanto na adolescência, como na fase adulta (American Psychiatric Association, 1994). Dessa forma, o conhecimento prático corrobora a visão científica da depressão e esclarece a importância desse sentimento no que se refere à identificação e ao manejo do quadro. Fato também verificado por Coutinho (2001) em estudo com crianças que apresentam a sintomatologia depressiva, nas quais a tristeza foi identificada como um tópico de extrema importância na apreensão das representações sociais da depressão.

A terceira subcategoria se refere à concepção psicossocial, a depressão como resultante da 24 insatisfação com as pessoas e o mundo. Os atores sociais evidenciam que as vicissitudes e contingências de suas vidas afetam sua forma de olhar e interpretar o mundo, o que indica que os sintomas da doença também afetam os indivíduos quanto ao nível da cognição.

Nessa subcategoria, percebeu-se que os dois grupos possuem representações consensuais, pois objetivaram a depressão no sentimento de insatisfação e não realização em face das adversidades do meio social que deixam os atores sociais impossibilitados de reagir aos estímulos hostis presentes no contexto sociocultural. Assim, a depressão é representada por vicissitudes que fogem ao controle do indivíduo e é vivenciada pelo conflito entre o desejo de solução do problema e a impossibilidade de concretizá-lo (Assumpção Jr \& Kucznski, 2001).

A última subcategoria referente à concepção/ descrição está ancorada nas manifestações físicoorgânicas objetivadas em doença, dor, remédios e no corpo. Aqui os sintomas da depressão são concebidos e descritos a partir de elementos funcionais do corpo, principalmente como uma expressão psicossomática, cujos resultados são corroborados pelas teorizações de Ballone (2001), segundo as quais a depressão manifestase por meio de sintomas psicossomáticos. Os aspectos físico-orgânicos inerentes às representações sociais da sintomatologia da depressão também podem ser observados em crianças e idosos (Coutinho, Gontiés, Araújo \& Sá, 2003).

No que tange à segunda categoria, referente às causas da depressão que foram ancoradas pelos atores sociais no campo das relações afetivas, verificou-se uma porcentagem de $52,7 \%$ das unidades temáticas e psicossociais com 47,3\%, o que remete à importância dos conflitos entre o indivíduo e o meio social e, principalmente, dos afetos na gênese de fatores que 
comprometem o bem-estar e a integridade física e psicológica (Tabela 2).

Os dados apresentados pelos adolescentes de escolas públicas revelam a seguinte disposição nas subcategorias: causas psicoafetivas $(46,5 \%)$ e psicossociais (53,5\%). Entre os adolescentes de escolas privadas, esses resultados revelam: psicoafetivas $(59,6 \%)$ e psicossociais $(40,4 \%)$ (Tabela 2). Observa-se que no primeiro grupo, a subcategoria de maior expressão foram as causas psicossociais, ao passo que o segundo grupo enfatizou as causas psicoafetivas.

Percebe-se que os grupos representam as causas psicoafetivas da depressão, principalmente, como uma conseqüência da perda de pessoas e objetos investidos de sentimentos, objetivadas através de frases e palavras que expressam a carência por afetos, e a sensação de ser incompreendido pelas pessoas:

... Você é apegada demais àquela coisa, de repente aquela coisa some da sua vida... baixa auto-estima... falta do carinho de quem se gosta... por que o namorado me deixou... eles só querem usar e depois jogam a gente fora... (G Pub).

... A sensação de perder alguém importante... falta de atenção e carinho ... falta de palavras... falta de alguém Ihe ouvir... ficar inseguro... a depressão dá quando a gente se sente incompreendido (G Priv).

Com relação à causa da depressão ancorada nos problemas psicossociais, as falas dos entrevistados evidenciam uma associação entre os sintomas da depressão e alguns problemas de ordem social e econômica específicos, como preconceito, desigualdade social, falta de dinheiro, de comida, de amigos, rejeição, e problemas no meio familiar.

\footnotetext{
... A depressão é causada por preconceito... não deveria existir o preconceito para não existir depressão... é causada pela desigualdade racial, social e emotiva... má convivência com os familiares e amigos falta comida... falta de companheirismo... pai bêbado. (G Pub).
}

... Essas são as causas principais: família e problemas... quando os amigos ficam evitando, ficam rejeitando... falta de amigos... quando você não tem seu grupo... devido a família que a pessoa tem... ao convívio com os pais... (G Priv).

Os jovens de escolas públicas tendem a enfatizar questões psicossociais mais concretas, próprias da realidade social menos favorecida na qual está inserida a sua pertença grupal, e níveis socioeconômico e cultural inferiores. A violência também é mais comum para esse grupo, porém para o grupo da escola privada as relações conflituosas inter e intragrupais se tornam mais latentes através da expressão de conteúdos que revelam a rejeição e não aceitação de certos indivíduos por parte de alguns grupos sociais estabelecidos no âmbito da escola. Os problemas com familiares são fortemente evocados na representação que os adolescentes constroem dos sintomas da depressão.

As questões concernentes ao surgimento de problemas relacionados à saúde mental na adolescência devem ser compreendidas a partir do paradigma que rege o desenvolvimento de nossa sociedade, assim o processo de exclusão social na adolescência é produto da marcante desigualdade de oportunidades existentes entre as classes sociais na realidade brasileira (Circe \& Melo, 2003).

A depressão possui, em sua etiologia, uma série de fatores que, segundo Coutinho (2001), podem estar relacionados a aspectos psicológicos, socioculturais e fisiológicos. Não é de espantar que o senso comum revele, no contexto social, o qual foi lócus desta investigação, problemas sociais e econômicos como os citados, visto que os fenômenos sociais e as manifestações individuais, sejam psicológicas ou de qualquer outra natureza, relacionam-se mutuamente, sendo, ambos, uma resultante de interações recíprocas.

Tabela 2. Distribuição da categoria causas da depressão, de suas subcategorias, das freqüências e percentual.

\begin{tabular}{|c|c|c|c|c|c|c|c|}
\hline \multirow{2}{*}{ Categoria } & \multirow{2}{*}{ Subcategorias } & \multicolumn{2}{|c|}{ G Pub } & \multicolumn{2}{|c|}{ G Priv } & \multicolumn{2}{|c|}{$\Sigma$} \\
\hline & & $f$ & $\%$ & $f$ & $\%$ & $f$ & $\%$ \\
\hline \multirow{2}{*}{ Causas da depressão } & Psicoafetivas & 27 & 46,5 & 31 & 59,6 & 58 & 52,7 \\
\hline & Psicossociais & 31 & 53,5 & 21 & 40,4 & 52 & 47,3 \\
\hline \multicolumn{2}{|c|}{ Total } & 58 & 100,0 & 52 & 100,0 & 110 & 100,0 \\
\hline
\end{tabular}


A terceira categoria refere-se ao tratamento da depressão. Os dados revelam que os adolescentes da rede pública de ensino ancoram o tratamento nas dimensões psicoafetiva (38,0\%), psicossocial $(23,5 \%)$, médica (22,0\%) e espiritual (16,5\%). Os adolescentes da escola privada associam o tratamento aos aspectos psicoafetivo (45,7\%), psicossocial (18,0\%), médico (23,7\%) e espiritual (12,7\%), (Tabela 3).

Verifica-se que a subcategoria tratamento psicoafetivo, para os jovens de ambas as instituições, obteve maior ênfase para a intervenção da sintomatologia da depressão. As falas dos adolescentes evidenciam a possibilidade de cura para a doença a partir dos afetos, ou seja, o sujeito deprimido precisa de um tratamento que priorize os aspectos afetivos e que considere os fatores psicológicos implicados, principalmente, dentro do meio familiar.

... melhora quem tem amor... ser amada por todo mundo... tem que dá atenção... tratar com carinho... deve ser tratado com conversa... levantar a autoestima... não pode ter problema ... a família tem que prestar atenção... (G Pub).

... na base do diálogo... algum tratamento psicológico... dá atenção pra pessoa... deve escutar a pessoa... entender o que ela tem... o interior da pessoa é o que vai ajudar... um psicólogo pra levantar a auto-estima... (G Priv).

Percebe-se ainda que a remissão dos sintomas da depressão pode se dar, também, para ambos os grupos, através do tratamento psicossocial, objetivado na convivência sadia com as pessoas investidas de afeto, na ajuda dos amigos, na aceitação social e, principal-mente, na ajuda dos familiares. Nessa subcategoria, há convergências e divergências no que concerne ao tratamento dos sintomas depressivos. Os jovens da escola pública enfatizam a ajuda dos amigos e a convivência social, ao passo que os jovens da escola privada ressaltam a necessidade de aceitação social e ajuda dos familiares.

Ambos os grupos consideraram o modelo médico como forma eficaz para o tratamento dos sintomas da depressão. Os atores sociais objetivaram essa subcategoria a partir de elementos como remédios, médico, hospital, internamento. É comum que os especialistas defendam a idéia de que o tratamento da depressão deva associar a psicoterapia ao tratamento medicamentoso. Segundo Angelotti (2001), é possível verificar uma maior eficácia quando se utilizam modalidades de tratamentos diferenciadas.

Os grupos apresentaram outra modalidade de tratamento, ancorada na dimensão espiritual. Esse é um exemplo de representações apoiadas em mitos e crenças no solo da cultura, transmitidas entre gerações (Moscovici, 2003), através das quais as ideologias e as instituições perduram seus valores, há tempos, nas sociedades. É válido salientar que esse componente espiritual foi verificado entre idosos, fato que independe da fronteira de idades (Coutinho, Gontiés, Araújo \& Sá, 2003).

A partir dos resultados, percebe-se que ambos os grupos expressam mais de uma modalidade de tratamento, corroborando as afirmações de Ballone (2001), que aponta que o tratamento dos sintomas depressivos deve se basear nos aspectos biológicos e psicossociais.

Evidenciou-se, neste estudo, que as representações sociais da sintomatologia depressiva elaboradas entre os adolescentes inseridos no contexto do ensino médio são construídas a partir do referencial de sua inserção psicoafetiva e socioeconômica, bem como pelos aspectos peculiares que permeiam a depressão na adolescência.

Tabela 3. Distribuição da categoria tratamento, de suas subcategorias, das freqüências e percentual.

\begin{tabular}{|c|c|c|c|c|c|c|c|}
\hline \multirow{2}{*}{ Categoria } & \multirow{2}{*}{ Subcategorias } & \multicolumn{2}{|c|}{ G Pub } & \multicolumn{2}{|c|}{ G Priv } & \multicolumn{2}{|c|}{$\Sigma$} \\
\hline & & $f$ & $\%$ & $f$ & $\%$ & $f$ & $\%$ \\
\hline \multirow{4}{*}{ Tratamento } & Psicoafetivo & 42 & 38,0 & 54 & 45,7 & 96 & 42,0 \\
\hline & Psicossocial & 26 & 23,5 & 21 & 18,0 & 47 & 20,5 \\
\hline & Médico & 24 & 22,0 & 28 & 23,7 & 52 & 23,0 \\
\hline & Espiritual & 18 & 16,5 & 15 & 12,7 & 33 & 14,5 \\
\hline \multicolumn{2}{|c|}{ Total } & 110 & 100,0 & 118 & 100,0 & 228 & 100,0 \\
\hline
\end{tabular}




\section{Conclusão}

Este trabalho versou sobre as representações sociais da sintomatologia depressiva no contexto do ensino médio, de forma comparativa entre escolas públicas e privadas. Os dados apreendidos entre os adolescentes possibilitaram representações consensuais e particularidades, de acordo com sua inserção sociocultural e pertença grupal.

Os aspectos apreendidos nessas representações, elaborados a partir dos adolescentes inseridos no contexto escolar, puderam ser encontrados empiricamente, não só por meio do discurso como fonte de dados relevantes, mas, também, por aspectos subjetivos observados e devidamente registrados durante todo o processo de investigação. Sinais de apatia, melancolia, fadiga, ansiedade, recusa e agressividade foram percebidos através das formas de comunicação oral e gestual.

Os grupos apresentaram convergências e divergências na elaboração das representações. As divergências mais significativas foram as relacionadas às causas da depressão. Os adolescentes da escola pública atribuíram a depressão a causas mais voltadas à dimensão psicossocial, objetivadas em fatores concretos oriundos das inibições sociais e econômicas; de forma diferente, os adolescentes da escola privada atribuíram as causas do problema com mais freqüência a dimensões afetivas e psicológicas.

Nesse sentido, as representações sociais elaboradas pelos atores sociais desta pesquisa revelaram uma similitude entre a concepção/ descrição representada pelos grupos e a descrita pela nosologia psiquiátrica, destacando uma série de sintomas, entre eles: solidão, isolamento, tristeza, desafeto, angústia, apatia, dor, insatisfação, negativismo, idéias suicidas, que remetem à depressão como doença e ao ser deprimido, indissociavelmente.

Pôde-se verificar, também, que, de acordo com o saber prático, a depressão é conseqüência de disfunções afetivas e de relações sociais insatisfatórias e patológicas, que perpetuam hábitos prejudiciais, principalmente, no âmbito da escola e da família. O tratamento da depressão foi ancorado, majoritariamente, nos afetos, o que remete a uma posição que priorize o estado psicológico do indivíduo através da atenção, do diálogo e da compreensão.
Um fato que merece atenção diz respeito às causas psicoafetivas que foram, significativamente, mencionadas, sobretudo, entre os adolescentes da rede privada. Infere-se que a ênfase nas mudanças no mercado de trabalho, no que diz respeito à exigência de qualificação e de dedicação exclusiva presente na sociedade atual, impede a manutenção das relações afetivas constantes, colocando em segundo plano os aspectos afetivos, em detrimento das relações laborais.

A partir dessas elucidações, pretende-se compreender o sofrimento provocado pela sintomatologia da depressão a partir da perspectiva das pessoas que sofrem com o problema em seu cotidiano, como, também, contribuir para o melhoramento na qualidade de vida e no bem-estar psicológico dos indivíduos inseridos no âmbito das instituições escolares que, muitas vezes, perpetuam hábitos excludentes e prejudiciais que podem refletir na manutenção da sintomatologia depressiva nessa fase do desenvolvimento, podendo se prolongar para a vida adulta.

\section{Referências}

American Psychiatric Association. (1994). Diagnostic and Statistical Manual of Mental Disorders - DSM-IV (4th ed.) Washington, DC: American Psychiatric Association.

Angelotti, G. (2001). Tratamento cognitivo-comportamental da depressão. In V. A. A. Camon (Org.). Depressão e psicossomática (pp.147-177). São Paulo: Pioneira Thomson Learning.

American Medical Association. (2002). Guia essencial de depressão. São Paulo: Aquariana.

Assumpção Jr., F. B., \& Kuczynski, E. (2001). Infância e adolescência. In R. Fráguas Jr. \& J. A. B. Figueiró. Depressões em medicina interna e em outras condições médicas: depressões secundárias (pp.387-399). Belo Horizonte: Atheneu.

Bahls, S. C. (2002a). Aspectos clínicos da depressão em crianças e adolescentes: clinical features. Jornal de Pediatria, 78 (5), 359-366.

Bahls, S. C. (2002b). Epidemiologia de sintomas depressivos em adolescentes de uma escola pública em Curitiba, Brasil. Revista Brasileira de Psiquiatria, 24 (2), 33-44.

Ballone, G. J. (2001). Depressão no idoso. Revisto em 2002. Acesso 24 fev. 2003, disponível em: www.psiqweb. med.br/geriat

Bardin, L. (2002). Análise de conteúdo. Lisboa: Edições 70.

Barbosa, G. A., Gaião e Barbosa, A. A., Dias, M. R., \& Di Lorenzo, W. C. (1995). Escala de Avaliação de Depressão em Crianças (CDRS-R): uma análise exploratória. Infanto-Revista Neuropsiquiatria da Infância e Adolescência, 5 (1), 19-25. 
Baron, P., \& Campbell, T. L. (1993) Gender differences in the expression of depressive symptoms in middle adolescents: an extension of early findings. Adolescence, 28 (112), 903-11.

Brent, D. A.(1993). Depression and suicide in children and adolescents. Pediatrics Review, 14 (10), 380-388.

Birmaher, B., Ryan, N. D., Williamson, D. E, Brent, D. A., Kaufman, J., \& Dahl, R. E. (1996). Childhood and adolescent depression: a review of the past 10 years. Part I. Journal of the American Academy of Child and Adolescent Psychiatry, 35 (11), 1427-1439.

Coutinho, M. P. L., Gontiés, B., Araújo, L. F., \& Sá, R. C. N. (2003). Depressão um sofrimento sem fronteira: representações sociais entre crianças e idosos. Psico-USF, 8 (2), 182-191.

Coutinho, M. P. L. (2001). Depressão infantil: uma abordagem psicossocial. João Pessoa: EdUFPB.

Circe, M. N. R., \& Mello, T. K. S. (2003). A política de atendimento à criança e ao adolescente no Brasil. In M. F. P. Alberto (Org.) Crianças e adolescentes que trabalham: cenas de uma realidade negada (pp.237-241). João Pessoa: EdUFPB.

Doise, W. (1990). Lês Représentations Sociales. In R. Ghiglione, C. Bonnet \& J. F. Richard (Orgs.). Traité de psychologie cognitive (v.3, pp.23-34). Paris: PUF.

Gunnell, D. J.(2000). The epidemiology of suicide. International Review of Psychiatry, 12(1), 21-26.

Feijó, R. B., Raupp, A. P. G., \& John, A. B. (1996). Eventos estressores de vida e sua relação com tentativas de suicídio em adolescentes. Jornal Brasileiro de Psiquiatria, 48(4), 151-157.

Jodelet, D. (2001).Representações sociais: um domínio em expansão. In D. Jodelet (Org.). As representações sociais (pp.17-44). Rio de Janeiro: EdUERJ.

Kazdin, A. E., \& Marciano, P. L. (1998). Childhood and adolescent depression. In E. Mash \& R. Barkley (Orgs.). Treatment of childhood disorders (pp.192-205). New York: The Guilford Press.

Kovács, M. J., \& Beck, A. T. (1977). An empirical-clinical approach toward a definition of childhood depression. In J. G. Schulterbrant \& A. Raskin. Depression in childhood; diagnosis, treatment and conceptual models (p.125). New York: Raven Press.
Kovács, M. J. (1985). The Children's depression innovatory (CDI). Psichopharmaco Bull, 21 (4), 955.

Martin, A., \& Cohen D. J. (2000). Adolescent depression: window of (missed?) opportunity editorial. Journal of the American Psychiatry, 157 (10), 1549-1551.

Moscovici, S. (1961). La psicanálise son image et son public. Paris: Press Universitaires de France.

Moscovici, S. (2003). Representações sociais: investigações em Psicologia Social. Petrópolis: Vozes.

Murray, C. J. L., \& Lopez, A. D. (1997). Global mortality, disability, and the contribution of risk factors: Global Burden of Disease study. Lancet, 349 (9063), 1436-1442.

Mirza, K., \& Michael, A. (1996). Major depression in children and adolescents. British Journal Hospital Medical, 55 (1-2), 57-61.

Olsson, G., \& von Knorring, A. L. (1999). Adolescent depression: prevalence in Swedish high-school students. Acta Psychiatrica Scandinavica, 99 (5), 324-331.

Roberts, R. E., Lewinsohn, P. M., \& Seeley, J. R. (1995). Symptoms of DSM-III-R Major Depression in Adolescence: Evidence from an Epidemiological Survey. Journal of the American Academy of Child and Adolescent Psychiatry, 34 (12), 1608-1617.

Sá, C. P. (1998). A Construção do objeto de pesquisa em representações sociais. Rio de Janeiro: EdUERJ.

Sadler, L. S. (1991). Depression in adolescents. Context, manifestations, and clinical management. Nursing Clinical North American, 26 (3), 559-572.

Walter, G. (1996).Depression in adolescence. Australian Family Physician, 25 (10), 1575-1582.

Widlöcher, D., \& Hardy, M. C. (1991). La Dépression. Paris: Overture Medicale.

Recebido em: 16/12/2004

Versão final reapresentada em: 22/6/2005

Aprovado em: 17/10/2005 